\title{
Fas Signaling in Dendritic Cells Mediates Th2 Polarization in HDM-Induced Allergic Pulmonary Inflammation
}

\begin{abstract}
Miaomiao Han ${ }^{1,2,3 t}$, Ran $\mathrm{Hu}^{2+}$, Jingyu $\mathrm{Ma}^{2}$, Baohua Zhang ${ }^{2}$, Ce Chen ${ }^{4}$, Huabin $\mathrm{Li}^{1 *}$, Jun Yang ${ }^{3 *}$ and Gonghua Huang ${ }^{2,4 *}$
\end{abstract}

${ }^{1}$ Department of Otolaryngology-Head and Neck Surgery, Center for Allergic and Inflammatory Diseases, Affiliated Eye and ENT Hospital, Fudan University, Shanghai, China, ${ }^{2}$ Shanghai Institute of Immunology, Shanghai Jiao Tong University School of Medicine, Shanghai, China, ${ }^{3}$ Xin Hua Hospital Affiliated to Shanghai Jiao Tong University School of Medicine, Shanghai, China, ${ }^{4}$ Guangdong Provincial Key Laboratory of Medical Molecular Diagnostics, Guangdong Medical University, Dongguan, China

\section{OPEN ACCESS}

Edited by:

Silvia Beatriz Boscardin, University of São Paulo, Brazil

Reviewed by: Yusei Ohshima,

University of Fukui, Japan Alexandre Castro Keller, Federal University of São Paulo, Brazil

*Correspondence: Huabin L allergyli@163.com

Jun Yang

yangjun@xinhuamed.com.cn Gonghua Huang gonghua.huang@shsmu.edu.cn

†These authors have contributed equally to this work

Specialty section:

This article was submitted to Antigen Presenting Cell Biology,

a section of the journal

Frontiers in Immunology

Received: 17 August 2018 Accepted: 10 December 2018 Published: 21 December 2018

Citation:

Han M, Hu R, Ma J, Zhang B, Chen C, Li H, Yang J and Huang $G$ (2018) Fas Signaling in Dendritic Cells

Mediates Th2 Polarization in

HDM-Induced Allergic Pulmonary Inflammation. Front. Immunol. 9:3045

doi: 10.3389/fimmu.2018.03045
Fas-Fas ligand (FasL) signaling plays an important role in the development of allergic inflammation, but the cellular and molecular mechanisms are still not well known. By using the bone marrow-derived dendritic cell (BMDC) transfer-induced pulmonary inflammation model, we found that house dust mite (HDM)-stimulated FAS-deficient BMDCs induced higher Th2-mediated allergic inflammation, associated with increased mucus production and eosinophilic inflammation. Moreover, FAS-deficient BMDCs promoted Th2 cell differentiation upon HDM stimulation in vitro. Compared to wild-type BMDCs, the Fas-deficient BMDCs had increased ERK activity and decreased IL-12 production upon HDM stimulation. Inhibition of ERK activity could largely increase IL-12 production, consequently restored the increased Th2 cytokine expression of OT-II CD4 ${ }^{+}$ $\mathrm{T}$ cells activated by Fas-deficient BMDCs. Thus, our results uncover an important role of DC-specific Fas signaling in Th2 differentiation and allergic inflammation, and modulation of Fas signaling in DCs may offer a useful strategy for the treatment of allergic inflammatory diseases.

\section{Keywords: allergic inflammation, dendritic cells, Fas, house dust mite, Th2}

\section{INTRODUCTION}

Allergic inflammation has been generally considered as a T helper (Th) 2-mediated chronic immune response (1). Th2 cells produce effector cytokines such as IL-4, IL-5, and IL-13 to mediate the respiratory symptoms. Among these effector cytokines, IL-4 is involved in IgE synthesis and IL-5 can drive eosinophilia in lung tissue, while IL-13 contributes to mucus overproduction, airway hyper-responsiveness (AHR), goblet cell metaplasia and airway remodeling (1-3). House dust mite (HDM) has been reported to cause $50-85 \%$ of allergic asthmatic inflammation (4). The polarization of a Th2-mediated immune response to inhaled allergens (such as HDM) is determined by the status of dendritic cells (DCs). DCs promote Th2 differentiation through upregulation of the expression of several costimulatory molecules such as CD86, OX40L and polarization cytokines including IL-6, IL-10, and IL-33 (5-8), as well as Th2-cell-attracting chemokines, such as CCL17 and CCL22 (9). Although accumulating evidence suggests that DCs are sufficient and necessary to 
initiate Th2 responses, the underlying signaling mechanism for DCs to direct Th2 differentiation and function is still not wellunderstood.

Fas (CD95, also named APO-1) signaling is widely considered to mediate apoptosis upon binding to its ligand (FasL, also called "CD95L or APO-1L") or its agonist antibody (10). In an ovalbumin (OVA)-induced mouse asthma model, Fas-deficient mice have delayed resolution of airway hyperresponsiveness (AHR) compared to wild-type mice (11). Further study indicates that Fas deficiency in $\mathrm{T}$ cells contributes to the prolonged resolution of airway inflammation (12). Recent studies have shown that Fas-FasL interaction could also activate nonapoptotic pathways, such as Fas signaling leading to $\mathrm{T}$ cell activation, proliferation and differentiation (13) and promoting Th17 polarization and Th17-mediated autoimmunity (14). Fasdeficient mice sensitized with OVA increase the expression of IL-4, IL-5, and IL-13 compared to wild-type mice (15). Although $\mathrm{DCs}$ are pivotal in regulating $\mathrm{T}$ cell activation, proliferation, differentiation and allergic inflammation, the role of Fas signaling in DCs in driving Th2 differentiation and Th2-mediated allergic inflammation still need to be elucidated.

In the present study, we used the well-established model by adoptively transferring HDM-pulsed BMDCs to recipient mice to explore the role of FAS signaling in DCs in pulmonary inflammation. We found that Fas deficiency in DCs led to increased mucus production, eosinophilic inflammation and Th2 response in vivo. Fas-deficient BMDCs promoted the production of Th2-related cytokines such as IL-4 and IL-13. Further mechanistic study showed that DCs directed Th2 differentiation by modulating the Fas-ERK-IL-12 axis. Collectively, our results identify an important signaling mechanism of DC-mediated Th2 responses and modulation of Fas signaling in DCs might offer a useful strategy for the treatment of eosinophilic lung inflammatory diseases.

\section{MATERIALS AND METHODS}

\section{Mice}

B6.MRL-Tnfrsf6 $6^{\text {pr }}$ mice were from The Jackson Laboratory. C57BL/6 mice were from Shanghai SLAC Laboratory Animal Center (Shanghai, China). All mice were kept in a specific pathogen-free (SPF) barrier facility maintained by Shanghai Jiao Tong University School of Medicine. All the experimental mice were used at 6-10 weeks. Animal protocols were approved by Institutional Animal Care and Use Committee of Shanghai Jiao Tong University School of Medicine.

\section{BMDC Culture}

BMDCs were cultured as previously described (16). Briefly, bone marrow cells were collected by perfusing mouse femur and tibia. After red blood cell lysis, cells were cultured in RPMI 1640 (Invitrogen Corp.) supplemented with 10\% fetal calf serum (FCS, Gibco), penicillin and streptomycin (Invitrogen), 2-mercaptoethanol (Sigma-Aldrich), supernatant from J5 cells (provided by Dr. Qibin Leng, Institut Pasteur of Shanghai, China) expressing GM-CSF (1:50) and $10 \mathrm{ng} / \mathrm{ml} \mathrm{IL-4} \mathrm{(R \& D).} \mathrm{On} \mathrm{day}$ 3 , the entire medium was removed and replaced with fresh differentiation medium. On day 7, the cells were harvested for analyses. The purity of CD11c ${ }^{+}$BMDCs was $>80 \%$.

\section{BMDC Adoptive Transfer Experiment}

In BMDC adoptive transfer experiment, wild-type or Fasdeficient BMDCs were pulsed with $50 \mu \mathrm{g} / \mathrm{ml}$ HDM (Greer Laboratories, Lenoir, NC) for $12 \mathrm{~h}$, washed, and then $1 \times$ $10^{6}$ HDM-pulsed BMDCs were administered intravenously into naïve C57BL/6 recipients. Recipients transferred with un-pulsed wild-type or Fas-deficient BMDCs as control. On day 1012 , all the recipients were lightly anesthetized and challenged intranasally with $10 \mu \mathrm{g} \mathrm{HDM}$ in $40 \mu \mathrm{l} \mathrm{PBS}$ and the mice were sacrificed for analysis on day 13 .

\section{Antibodies and Flow Cytometry}

Anti-mouse CD11c (N418), MHC-II (M5/114.15.2), CD11b (M1/70), Ly6G (RB6-8C5), siglec F (E50-2440), CD4 (RM45), TCR $\beta$ (H57-597), IL-4 (11B11), IL-13 (eBio13A), IL-17A (eBio17B7), CD25 (PC61.5), CD44 (IM7), CD62L (MEL-14), CD69 (H1.2F3), Ki-67 (SolA15), IL-12p40 (C17.8), CD45 (30F11), CD178 (MFL3) antibodies were obtained from eBioscience. Anti-mouse IL-5 (TRFK5) was obtained from BD Biosciences. Anti-mouse CCR3 (J073E5) was obtained from Biolegend. For surface staining, cells were stained with antibodies in PBS containing 1\% FCS (Hyclone) on ice for $30 \mathrm{~min}$. For intracellular staining, cells were stimulated with PMA (SigmaAldrich) and ionomycin (Sigma-Aldrich) for $5 \mathrm{~h}$ in the presence of Golgistop (BD Biosciences) before being stained according to the manufacturer's instructions (eBioscience). $\mathrm{CD} 4^{+} \mathrm{T}$ cell proliferation was detected by anti-Ki-67 staining according to the manufacturer's instructions (eBioscience) 3 days after co-culture. Labeling OTII CD4 ${ }^{+} \mathrm{T}$ cells with CFSE (carboxyfluorescein diacetate succinimidyl diester; Invitrogen), cell proliferation was detected by flow cytometry 4 days after co-culture. For cell apoptosis analysis, cells were stained with CaspACE ${ }^{\mathrm{TM}}$ FITCVAD-FMK in situ Marker (Promega). The samples were acquired on a FACSCantoII (BD) or LSRFortessa ${ }^{\mathrm{TM}} \mathrm{X}-20$ (BD) and analyzed with FlowJo software (Treestar).

\section{Bronchoalveolar Lavage Fluid (BALF) Collection and Lung Mononuclear Cell Isolation}

For BALF collection, lung tissues were lavaged with $1 \mathrm{ml}$ cold PBS for 3 times and the supernatant was collected. Lung mononuclear cells were prepared as previously described (17). Briefly, lung tissues were removed, minced and digested with $1 \mathrm{mg} / \mathrm{ml}$ Collagenase IV (Life Technologies) in RPMI-1640 (Hyclone) with 5\% FCS (Hyclone) for $45 \mathrm{~min}$ at $37^{\circ} \mathrm{C}$. Cells were enriched by using $38 \%$ Percoll gradient (GE Healthcare Life Sciences). Red blood cells were lyzed with ACK lysis buffer (R\&D Systems). Cells were harvested for analyses.

\section{Histology}

Left lobe of lung tissues were removed from mice after BALF collection, fixed with $4 \%$ paraformaldehyde (PFA) at room temperature for $24 \mathrm{~h}$ and embedded in paraffin, cut into $5-\mu \mathrm{m}$ sections for hematoxylin and eosin (HE) or periodic acid-Schiff 
(PAS) staining. The lung inflammation was blindly quantified using HE-stained sections according to the criteria previously published (18). The quantification of the goblet cell hyperplasia in the airway was done as previously described (19).

\section{RNA Isolation and Quantitative PCR (qPCR)}

Total RNAs of lung tissues were isolated using Trizol regent (Invitrogen). Total RNAs of cells were isolated using RNeasy mini Kit (QIAGEN) according to the manufacturer's instructions. $1 \mu \mathrm{g}$ total RNA was used for reverse transcription with PrimeScript RT Master Mix (TAKARA) according to the manufacturer's instructions in a total volume of $20 \mu \mathrm{l}$. qPCR was carried out with SYBR Green PCR Master Mix (Applied Biosystems) in a Vii7 Real-Time PCR system (Applied Biosystems). mRNA expression of genes was normalized to Hprt. The primers shown below were from Primerbank: Hprt, forward primer: TCAGTCAACGGGGGACATAAA, reverse primer: GGGGCT GTACTGCTTAACCAG; Il4, forward primer: GGTCTCAAC CC CCAGCTAGT, reverse primer: GCCGATGATCTCTCT CAAGTGAT; $I l 17 a$, forward primer: TCAGCGTGTCCAAAC ACTGAG, reverse primer: CGCCAAGGGAG TTAAAGACTT; Ifng, forward primer: GCCACGGCACAGTCATTGA, reverse primer: TGCTGATGGCCTGATTGTCTT; Il6, forward primer: CTGCAAGAGACTTCCATCCAG, reverse primer: AGTGGT ATAGACAGGTATGTTGG; Il12p35, forward primer: CAATCA CGC TACCTCCTCTTT, reverse primer: CAGCAGTGCAGG AATAATGTTTC; Il12p40, forward primer: GTCCTCAGAAGC TAACCATCTC, reverse primer: CCAGAGC CTATGACTCCA TGTC; Il10, forward primer: CTTACTGACTGGCATGAGGAT CA, reverse prime: GCAGCTCTAGGAGCATGTGG. The other primers were used as described, such as Il5, Il13 (20), Gata3 (21), Il9 (22), Tnfsf4 (8), Cd86 (23), Tslp (24).

\section{Cell Stimulation and Culture}

BMDCs were stimulated with HDM in the presence or absence of Fas agonistic antibody Jo2 $(1 \mu \mathrm{g} / \mathrm{ml}, \mathrm{BD})$ or Fas antagonistic antibody kp7-6 (1 mM, Merk) for $5 \mathrm{~h}$ for RNA analysis. For drug inhibitor treatments, cells were incubated with vehicle (DMSO) or U0126 $(10 \mu \mathrm{M})$ (from Calbiochem) for $0.5-1 \mathrm{~h}$ before adding other stimuli. For BMDC-T cell co-culture, BMDCs and flow cytometry-sorted naïve OT-II CD4 ${ }^{+} \mathrm{T}$ cells $\left(\mathrm{CD} 4{ }^{+} 25^{-} \mathrm{CD} 44^{-} \mathrm{CD}_{2} \mathrm{~L}^{+}\right.$, purity $\left.>99 \%\right)$ were mixed at a ratio of 1:10 in the presence of $\mathrm{OVA}_{323-339}$ peptide and HDM, and then the $\mathrm{CD}^{+}{ }^{+} \mathrm{T}$ cells were harvested at $48 \mathrm{~h}$ for mRNA analysis or supernatant was harvested at $72 \mathrm{~h}$ for ELISA. For cytokine treatment, cultures were supplemented with $1 \mathrm{ng} / \mathrm{ml}$ IL-12p70 $(\mathrm{R} \& \mathrm{D})$.

\section{Protein Analysis}

Concentrations of IL-4 and IL-13 were measured by ELISA according to the manufacturer's instructions (R\&D; eBioscience). Read the OD values at $450 \mathrm{~nm}$ on the MultiSKAN GO microplate reader (Thermo). Immunoblot analysis was performed as described (25) with antibody to ERK phosphorylated at Thr202 and Tyr204, antibody to p38 phosphorylated at Thr180 and Tyr182, antibody to JNK phosphorylated at Thr183 and Tyr185 and antibody to ERK (all from Cell Signaling Technology), antibody to alpha tubulin (Proteintech).

\section{Statistical Analysis}

All statistical analyses were performed by unpaired Student's $t$ tests or ANOVA using GraphPad Prism software (version 5.0). $P<0.05$ was considered significant. Results represent means \pm SEM.

\section{RESULTS}

\section{Fas-Deficient BMDCs Enhance HDM-Induced Pulmonary Inflammation}

To explore the role of Fas signaling in DCs in the regulation of HDM-induced allergic inflammation in mice, we used a BMDC adoptive transfer protocol to induce lung inflammation (Figure 1A). We transferred HDM-pulsed or un-pulsed wildtype or Fas-deficient BMDCs into naïve wild-type recipient mice. After HDM re-challenged, mice received HDM-pulsed BMDCs showed higher total cell number in the bronchoalveolar lavage (BAL) compared to mice received un-pulsed BMDCs (Figure 1B). A significantly increased total cell number was also observed in the BAL of recipients transferred with HDM-pulsed Fas-deficient BMDCs (Figure 1B). We also observed higher inflammatory cell infiltration and mucus production in lung tissues of recipients transferred with HDM-pulsed Fas-deficient BMDCs than those transferred with HDM-pulsed wild-type BMDCs (Figures 1C,D). Flow cytometry showed that a dramatically increased eosinophil infiltration both in the BAL and lung tissues of recipients transferred with HDMpulsed Fas-deficient BMDCs compared to those transferred with HDM-pulsed wild-type BMDCs (Supplementary Figures 1A,B). We also analyzed the inflammatory eosinophils (iEos) $\left(\mathrm{CD} 45^{+}\right.$Siglec $\left.\mathrm{F}^{\text {int }} \mathrm{CCR}^{+}{ }^{+} \mathrm{CD} 2 \mathrm{~L}^{-}\right)$and resident eosinophils (rEos) $\left(\mathrm{CD} 5^{+}\right.$Siglec $\left.\mathrm{F}^{\text {int }} \mathrm{CCR}{ }^{+} \mathrm{CD} 2 \mathrm{~L}^{+}\right)$in lung tissues (26). The cell number of iEos was increased in recipients transferred with HDM-pulsed Fas-deficient BMDCs compared to those transferred with HDM-pulsed wild-type BMDCs, but rEos was comparable between recipients transferred with HDM-pulsed wild-type BMDCs and those transferred with Fas-deficient BMDCs (Figure 1E). However, the neutrophil infiltration had no difference between the two groups (Supplementary Figures 1C,D). Together, these data indicate that HDM-pulsed Fas-deficient BMDCs can induce more severe allergic airway inflammation than HDM-pulsed wild-type BMDCs.

\section{Fas Signaling in BMDCs Does Not Affect $\mathrm{CD4}^{+} \mathrm{T}$ Cell Proliferation, Apoptosis, and Activation in vivo}

In addition to a role of innate immune cells in the development of allergic inflammation, the adaptive immune system also play an important role in driving and sustaining this inflammation (27). A comparable $\mathrm{CD}^{+}{ }^{+} \mathrm{T}$ cell activation was observed in lung tissues of the recipients transferred with HDM-pulsed wild-type or Fas-deficient BMDCs (Figure 2A). We also 


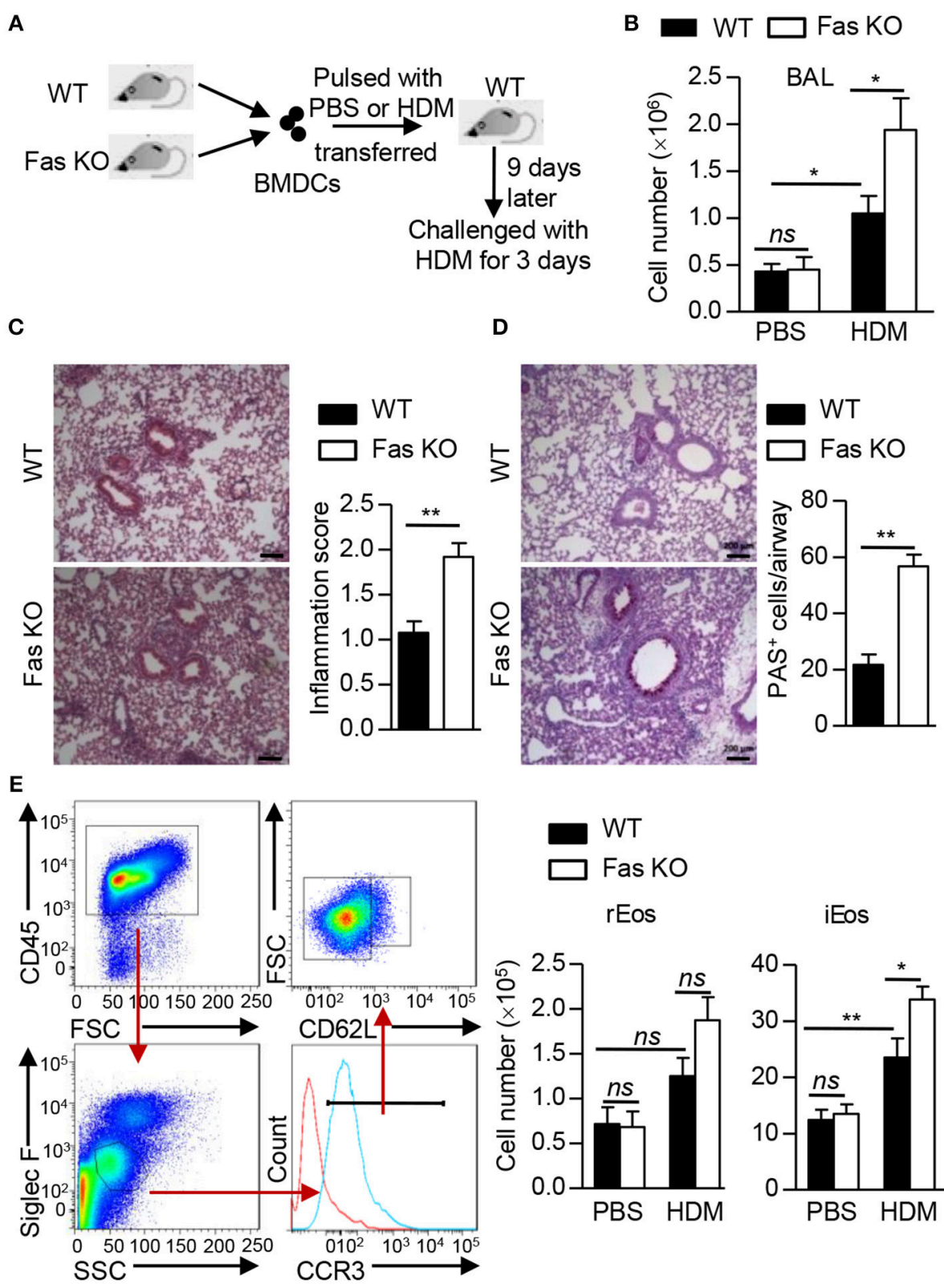

FIGURE 1 | Fas-deficient BMDCs enhance HDM-induced pulmonary inflammation. (A) Protocol for HDM-induced allergic airway inflammation using BMDC transfer experiment. (B) Total cell number in the BAL. (C) HE staining of lung sections from recipients transferred with HDM-pulsed wild-type or Fas-deficient BMDCs and quantitative analysis. Scale Bars represent $200 \mu \mathrm{m}$. (D) PAS staining of lung sections from recipients transferred with HDM-pulsed wild-type or Fas-deficient BMDCs and quantification of PAS ${ }^{+}$cells in airways. Scale Bars represent $200 \mu \mathrm{m}$. (E) Gating strategy and cell number of inflammatory eosinophils (iEos)

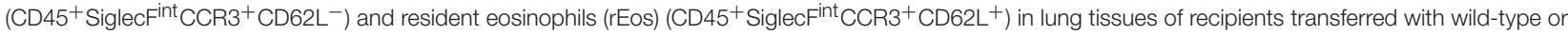
Fas-deficient BMDCs pulsed or un-pulsed with HDM. ${ }^{*} P<0.05$, ${ }^{\star \star} P<0.01$, ns, not significant. Data are representative of two independent experiments with $5-8$ mice per group (B-E). Student's $t$-tests $\mathbf{( C , D )}$ or two-way ANOVA $\mathbf{( B , E )}$ were performed and data were presented as mean \pm SEM.

examined whether Fas-deficient in BMDCs could affect CD4 ${ }^{+}$ $\mathrm{T}$ cell proliferation or apoptosis in vivo, we performed Ki67 and VAD staining assay, respectively. Our results showed that the proportion of $\mathrm{Ki}-67^{+} \mathrm{CD} 4^{+} \mathrm{T}$ cells and the median fluorescence intensity (MFI) value of $\mathrm{VAD}^{+} \mathrm{CD} 4^{+} \mathrm{T}$ cell in lung tissues and mediastinal lymph nodes $(\mathrm{mLN})$ had no differences between the recipients transferred with HDMpulsed wild-type BMDCs and those transferred with Fasdeficient BMDCs (Figures 2B-E). Taken together, these results show that Fas signaling in BMDCs is not required for $\mathrm{CD} 4^{+}$ $\mathrm{T}$ cell activation, proliferation and apoptosis upon HDM treatment. 
A

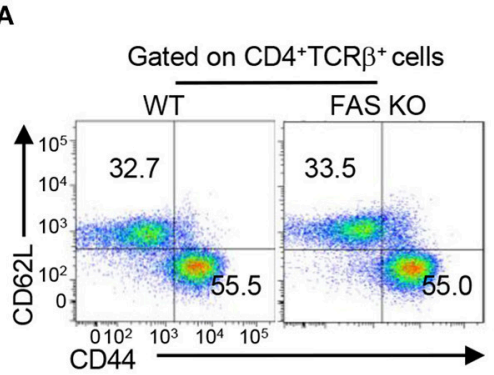

C

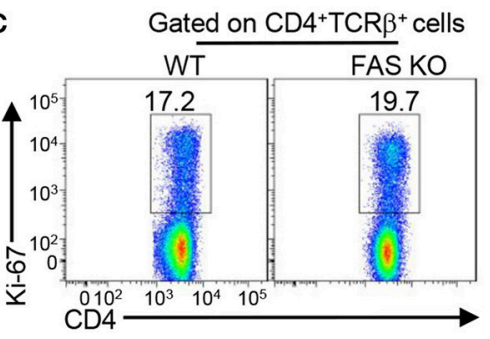

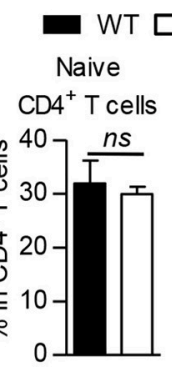
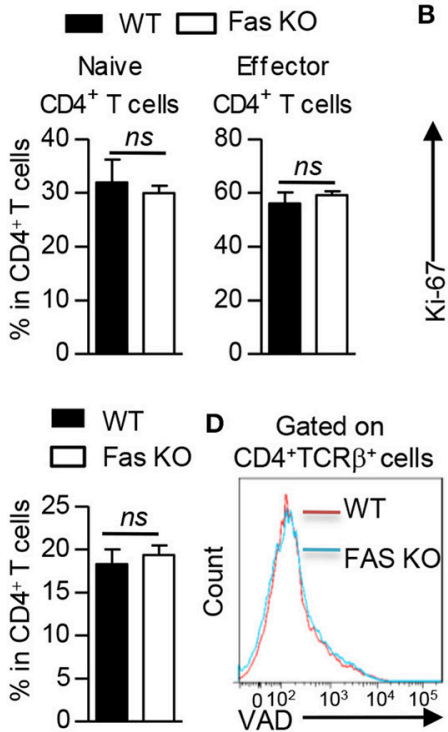

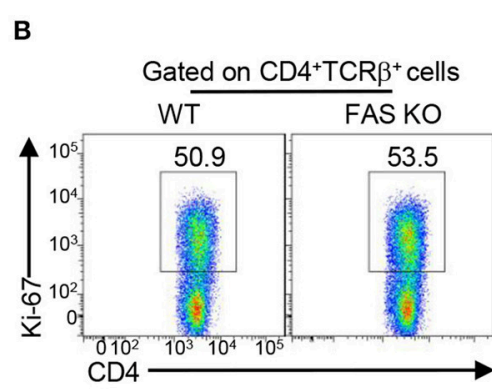

WT
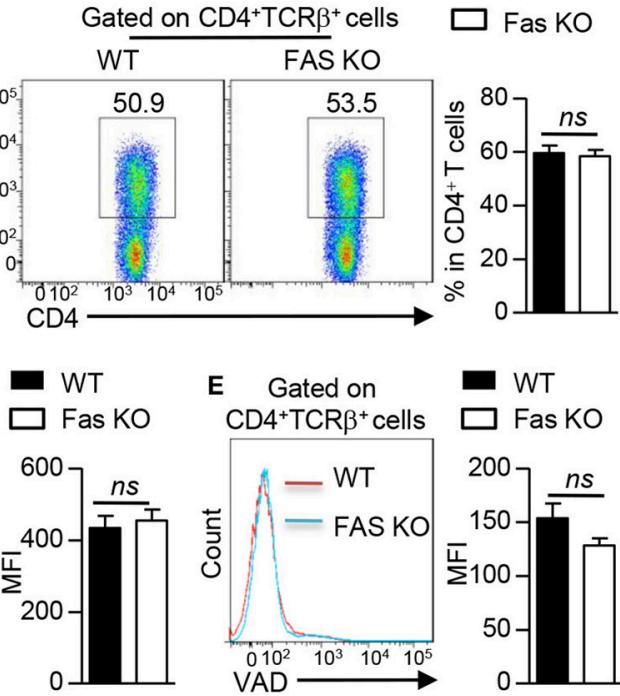

FIGURE 2 | Fas signaling in BMDCs does not affect CD4 ${ }^{+} \mathrm{T}$ cell proliferation, apoptosis and activation in vivo. Mice were treated as Figure 1A described. (A) Flow cytometry (left) and proportions (right) of naïve $\mathrm{CD} 4^{+} \mathrm{T}$ cells $\left(\mathrm{CD} 4^{+} \mathrm{TCR} \beta^{+} \mathrm{CD} 62 \mathrm{~L}^{+} \mathrm{CD} 44^{-}\right)$and effector $\mathrm{CD} 4^{+} \mathrm{T}$ cells $\left(\mathrm{CD} 4^{+} \mathrm{TCR}^{+}{ }^{+} \mathrm{CD} 62 \mathrm{~L}^{-} \mathrm{CD} 44^{+}\right)$in lung tissues. (B,C) Flow cytometry (left) and proportion (right) of Ki-67 expression in CD4 ${ }^{+}$T cells of lung tissues (B) and mLN (C). (D,E) Flow cytometry (left) and Median Fluorescence Intensity (MFI) of VAD (right) staining in CD4+ $\mathrm{T}$ cells of lung tissues (D) and $\mathrm{mLN}$ (E). $n s$, not significant. Data are representative of two independent experiments with 4-6 mice per group (A-E). Student's $t$-tests (A-E) were performed and data were presented as mean \pm SEM.

\section{Fas-Deficient BMDCs Promote Th2 Responses Upon HDM Treatment}

Given that there was no difference in the proliferation and apoptosis of $\mathrm{CD}^{+} \mathrm{T}$ cells in the recipients transferred with HDM-pulsed wild-type or Fas-deficient BMDCs, we reasoned that the difference in inflammatory response might be due to the potential of these cells to produce inflammatory cytokines. Thus, we first measured cytokine expression in lung tissues of these two group mice. The qPCR results showed that the recipients transferred with HDM-pulsed Fas-deficient BMDCs had higher mRNA expression of Il4 (encoding IL-4), Il5 (encoding IL-5), and Il13 (encoding IL-13), but comparable expression of Ifng (encoding IFN $\gamma$ ) and Il17a (encoding IL-17A) in lung tissues than those transferred with HDM-pulsed wildtype BMDCs (Figure 3A). Although a comparable percentage and cell number of $\mathrm{CD}^{+} \mathrm{T}$ cells was observed in lung tissues of the two HDM-pulsed groups (Figure 3B), intracellular staining showed that recipients transferred with HDM-pulsed Fas-deficient BMDCs had higher percentage of IL- $4^{+}$, IL- $5^{+}$, and $\mathrm{IL}-13^{+} \mathrm{CD} 4^{+} \mathrm{T}$ cells in lung tissues than those transferred with wild-type BMDCs, along with higher cell number of IL$4^{+}, \mathrm{IL}^{-} 5^{+}$, and IL- $13^{+} \mathrm{CD} 4^{+}$T cells (Figures $3 \mathrm{C}, \mathrm{D}$ ), whereas the percentage and cell number of $\mathrm{IL}-17^{+} \mathrm{CD} 4^{+} \mathrm{T}$ cells were similar in the two groups (Supplementary Figures 2A,B). However, the percentage and cell number of $\mathrm{IL}-4^{+}, \mathrm{IL}-5^{+}$, and IL$13^{+} \mathrm{CD} 4^{+} \mathrm{T}$ cells had no difference between the recipients transferred with un-pulsed wild-type and Fas-deficient BMDCs (Supplementary Figures 2C,D). Taken together, these results demonstrate that HDM-pulsed Fas-deficient BMDCs promote Th2 responses in vivo.

\section{Fas Signaling in BMDCs Instructs Th2 Cell Differentiation in vitro}

To determine whether the increased Th2 response in vivo is due to the direct interaction between DCs and T cells, we cocultured wild-type or Fas-deficient BMDCs with naïve OT-II $\mathrm{CD}^{+} \mathrm{T}$ cells in the presence of $\mathrm{OVA}_{323-339}$ peptide with or without HDM. The polarization of $\mathrm{CD} 4^{+} \mathrm{T}$ cells was determined by qPCR or ELISA, respectively. OT-II CD4 ${ }^{+} \mathrm{T}$ cells activated with HDM-stimulated Fas-deficient BMDCs had higher IL-4 and IL-13 expression both in mRNA and in protein levels than those stimulated with wild-type BMDCs (Figures 4A,B). GATA3, the master transcription factor for Th2 cell differentiation, was also found increased in T cells activated by HDM-pulsed Fas-deficient BMDCs, while the expression of Il9 and Ill0 was comparable (Figure 4A). The activation and proliferation of OT-II CD4 $4^{+} \mathrm{T}$ cells activated by HDM-pulsed both wild-type and Fas-deficient BMDCs were comparable (Figures 4C-E). Collectively, these data indicate that Fas signaling mediates the direct crosstalk between BMDCs and Th2 cells upon HDM stimulation in vitro.

\section{Fas-Deficient BMDCs Promote Th2 Differentiation by Inhibiting IL-12 Expression}

Next we explored the molecular mechanism by which Fas signaling in BMDCs to shape Th2 differentiation upon HDM stimulation. We stimulated wild-type BMDCs with Fas agonistic antibody Jo2, the expression of Cd86, Tslp, Il10, Il6, and Tnfsf4, which had been reported to regulate Th2 polarization, had no difference compared to control group 

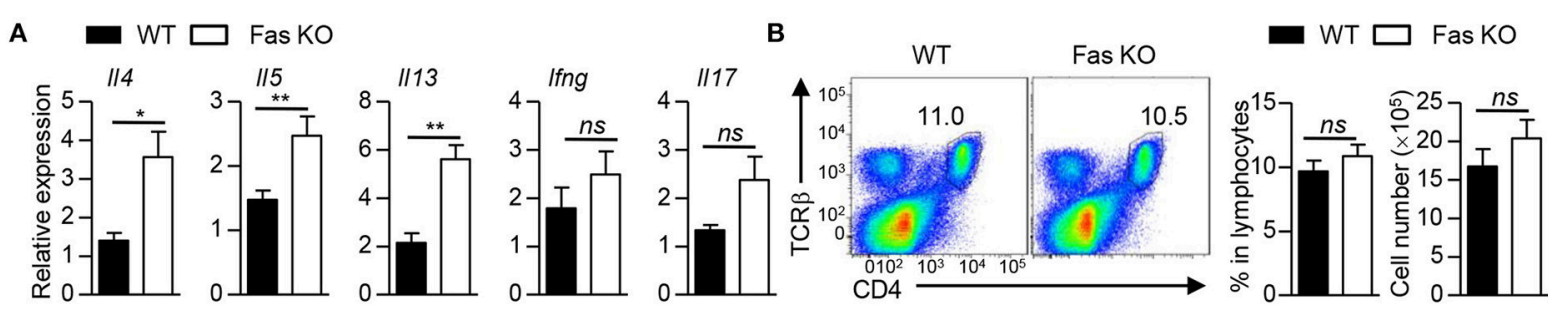

C Gated on $\mathrm{CD} 4^{+} \mathrm{TCR} \beta^{+}$cells
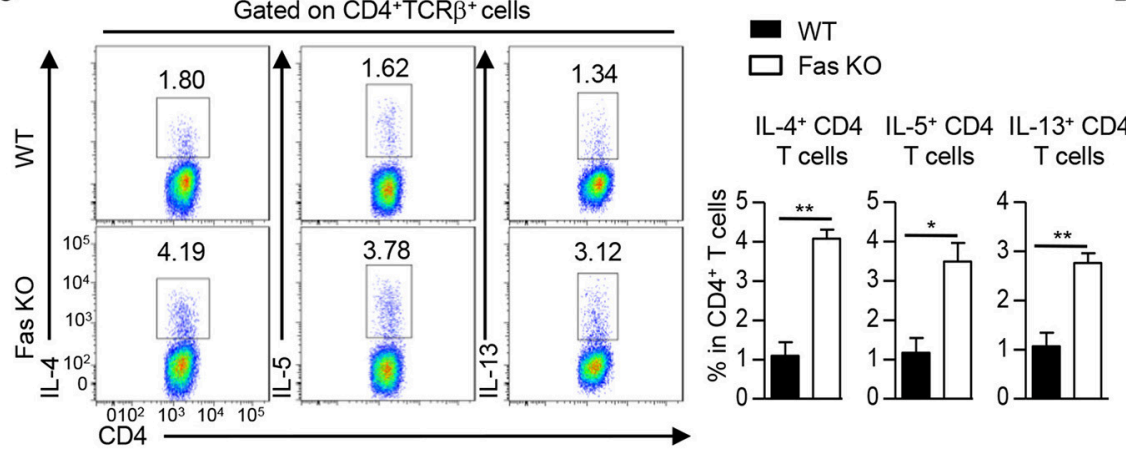

D

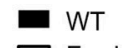

$\square$ Fas KO
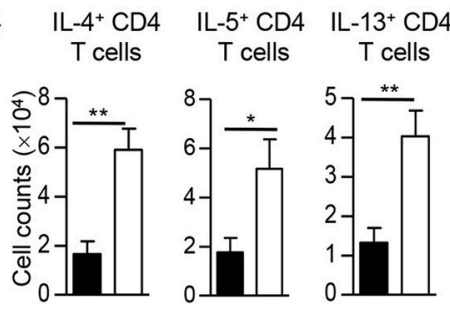

FIGURE 3 | Fas-deficient BMDCs promote Th2 responses upon HDM treatment. Mice were treated as Figure 1A described. (A) mRNA expression of II4, II5, II13, Ifng, and I/17 in lung tissues was detected by qPCR and normalized to Hprt. (B) Flow cytometry (left), percentage (middle), and cell number (right) of CD4+ T cells in

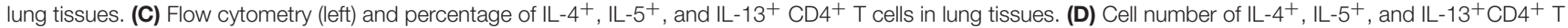
cells in lung tissues. ${ }^{*} P<0.05,{ }^{* *} P<0.01, n s$, not significant. Data are representative of two independent experiments with $4-5$ mice (A-D) per group. Student's $t$-tests (A-D) were performed and data were presented as mean \pm SEM.

(Figure 5A and Supplementary Figure 3A). HDM stimulation could dramatically increase the expression of Il6 and Tnfsf4, while Fas agonistic antibody Jo2 did not affect the expression of these two genes (Figure 5A), indicating that FAS signaling is not required for Il6 and Tnfsf4 expression in DCs upon HDM stimulation. IL-12 has been reported to affect Th2 differentiation (28). Fas agonistic antibody Jo2 stimulation could not affect the expression of Il12p35 and Il12p40 in wild-type BMDCs without HDM stimulation (Figure 5A). Upon HDM stimulation, the expression of Il12p35 and Il12p40 was significantly increased compared with non-HDM stimulation, and Fas agonistic antibody Jo2 could further enhance the expression of Il12p35 and Il12p40 in mRNA level and IL-12p70 in protein level (Figures 5A,B). Given that HDM-stimulated BMDCs had increased FasL, Fas antagonistic antibody kp7-6-treated HDMpulsed wild-type BMDCs produced lower Il12p40 in mRNA level and IL-12p70 in protein level than those of BMDCs stimulated with HDM alone, but the expression of Il12p35 had no difference between HDM and HDM plus kp7-6 stimulated BMDCs (Figures 5C,D and Supplementary Figure 3B). Fasdeficient BMDCs had decreased expression of Il12p35 and Il12p40 compared to wild-type BMDCs upon HDM stimulation in the presence of Fas agonistic antibody Jo2 (Figure 5E). We next examined whether the lower expression of IL-12 in Fas-deficient BMDCs could contribute to the increased Th2 differentiation. We added exogenous IL-12 into the BMDC-T cell co-culture system and found that the IL-12 supplement could significantly decrease the expression of Th2-related cytokines, such as IL- 4 and IL-13 in OT-II CD $4^{+} \mathrm{T}$ cells activated by Fas-deficient BMDCs. The expression of IFN $\gamma$ and IL-17 was comparable between wild-type and Fas-deficient BMDC activated $\mathrm{T}$ cells (Figure 5F). Altogether, these results indicate that Fas-deficient BMDCs promote Th2 differentiation through downregulation of IL-12 expression.

\section{Fas Signaling Regulates IL-12 Expression by Modulation of ERK Activation in BMDCs}

We next examined the downstream signaling of Fas involved in the regulation of IL-12 expression by analyzing the activation of p38, JNK, and ERK in BMDCs. We found that the phosphorylation of ERK was decreased in wild-type BMDCs treated with Fas agonistic antibody Jo2 and HDM compared to those treated with HDM alone (Figure 6A). Accordingly, the activation of ERK in Fas-deficient BMDCs was increased compared to that of wild-type BMDCs upon HDM and Fas agonistic antibody Jo2 stimulation (Figure 6B). To determine whether the increased ERK activation was contributed to the decreased IL-12 expression in Fas-deficient BMDCs, we treated Fas-deficient BMDCs with specific ERK inhibitor U0126, which resulted in a dramatically increased IL-12p70 expression in Fasdeficient BMDCs (Figure 6C). Consequently, U0126-treated Fasdeficient BMDCs completely restored the increased IL-4 and IL-13 expression in $\mathrm{T}$ cells to the level of $\mathrm{T}$ cells activated with wild-type BMDCs (Figure 6D). These results indicate that Fas signaling regulates IL-12 expression and Th2 differentiation through modulating the ERK activity in BMDCs. 


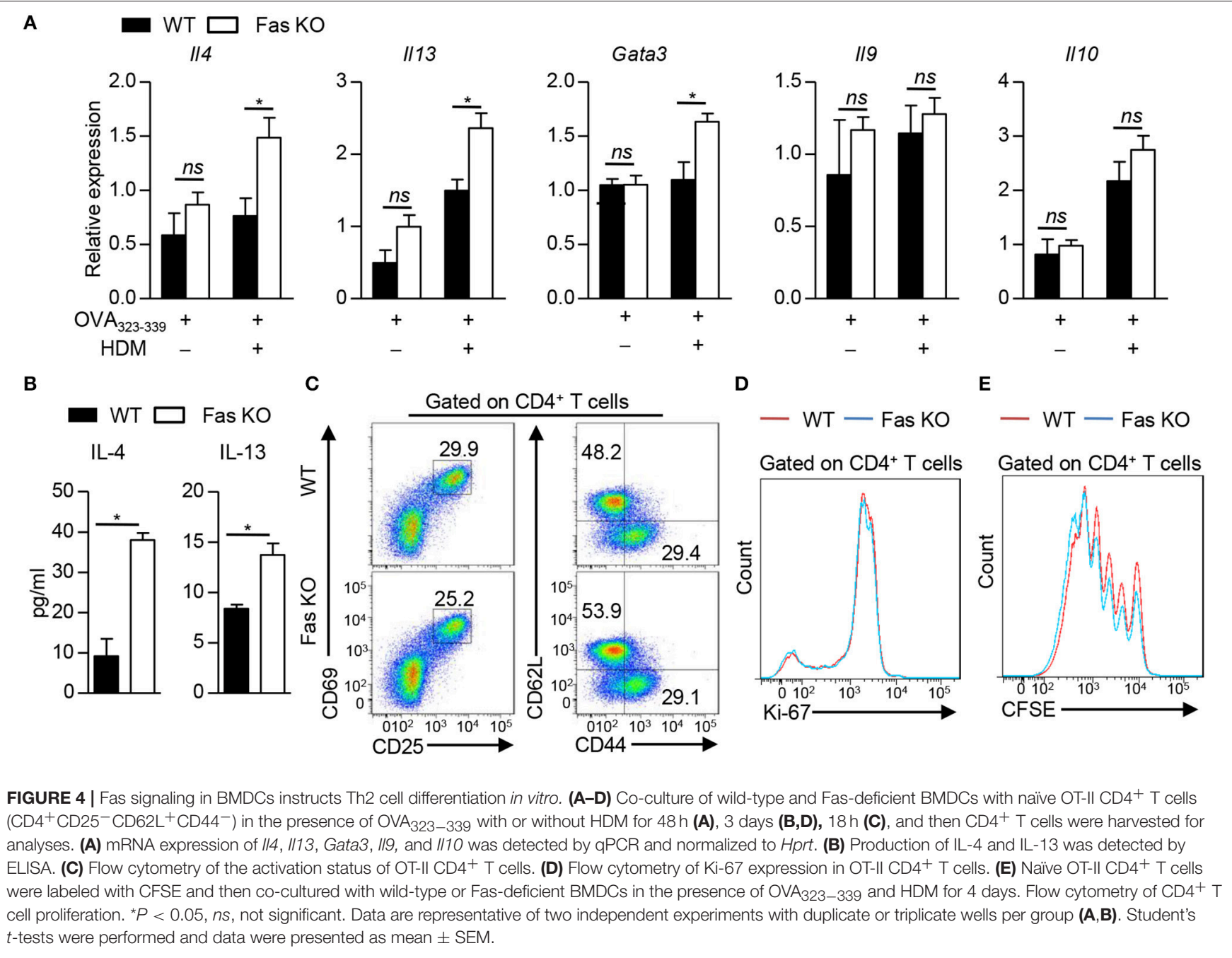

\section{DISCUSSION}

Numerous studies have reported that administration of allergenpulsed DCs is sufficient to induce airway inflammation by polarizing Th2 responses (3, 29-31). However, the mechanism of DCs to regulate Th2 cell differentiation is still unclear (32). In this study, we used a BMDC-transfer protocol to investigate the important role of DC-specific Fas signaling in the pathogenesis of HDM-induced Th2-mediated allergic inflammation. We found that HDM-pulsed Fas-deficient BMDCs could promote Th2 responses and allergic eosinophilic inflammation, without affecting $\mathrm{T}$ cell apoptosis and proliferation in the recipients. Our study identified a crucial role of Fas signaling in regulating IL12 expression by modulating ERK activity in DCs to direct Th2 differentiation upon HDM stimulation, which may provide an attractive treatment strategy for allergic diseases.

DCs constitutively express non-canonical costimulatory molecule Fas (13), which is activated by FasL or Fas agonistic antibody Jo2 to induce an apoptotic signaling. Numerous studies have shown that activated Fas signaling in DCs induces the secretion of cytokines such as IL-1 $\beta$ and CXC or CC chemokines $(13,33,34)$, which may play important roles in the recruitment, activation and proliferation of naïve $\mathrm{T}$ cells $(14,35)$. FasFasL interaction on $\mathrm{T}$ cells has been proposed to promote the differentiation of naïve $\mathrm{T}$ cells into functional $\mathrm{T}$ cells (14), but little is known about how the Fas signaling in DCs regulating Th2 differentiation. DCs could promote Th2 differentiation through upregulation signal 2 (such as CD86, OX40L) $(7,36,37)$ or signal 3 (such as IL-6, IL-10,TSLP) (38-40). In this study, we found that Fas agonistic antibody Jo2 stimulation did not affect the expression of OX40L and IL-6. However, Jo2 stimulation increased IL-12 expression in BMDCs stimulated with HDM. Accordingly, the ablation of Fas in DCs largely reduced the expression of IL-12, which contributed to the increased Th2 differentiation. Our results identify a new mechanism by which DC regulating Th2 responses through modulation of IL-12 production during inflammation development.

ERK signaling has been shown to play important roles in Fas-mediated non-apoptotic function. Ligation of Fas agonistic antibody Jo2 with Fas on DCs can promote the activation of 


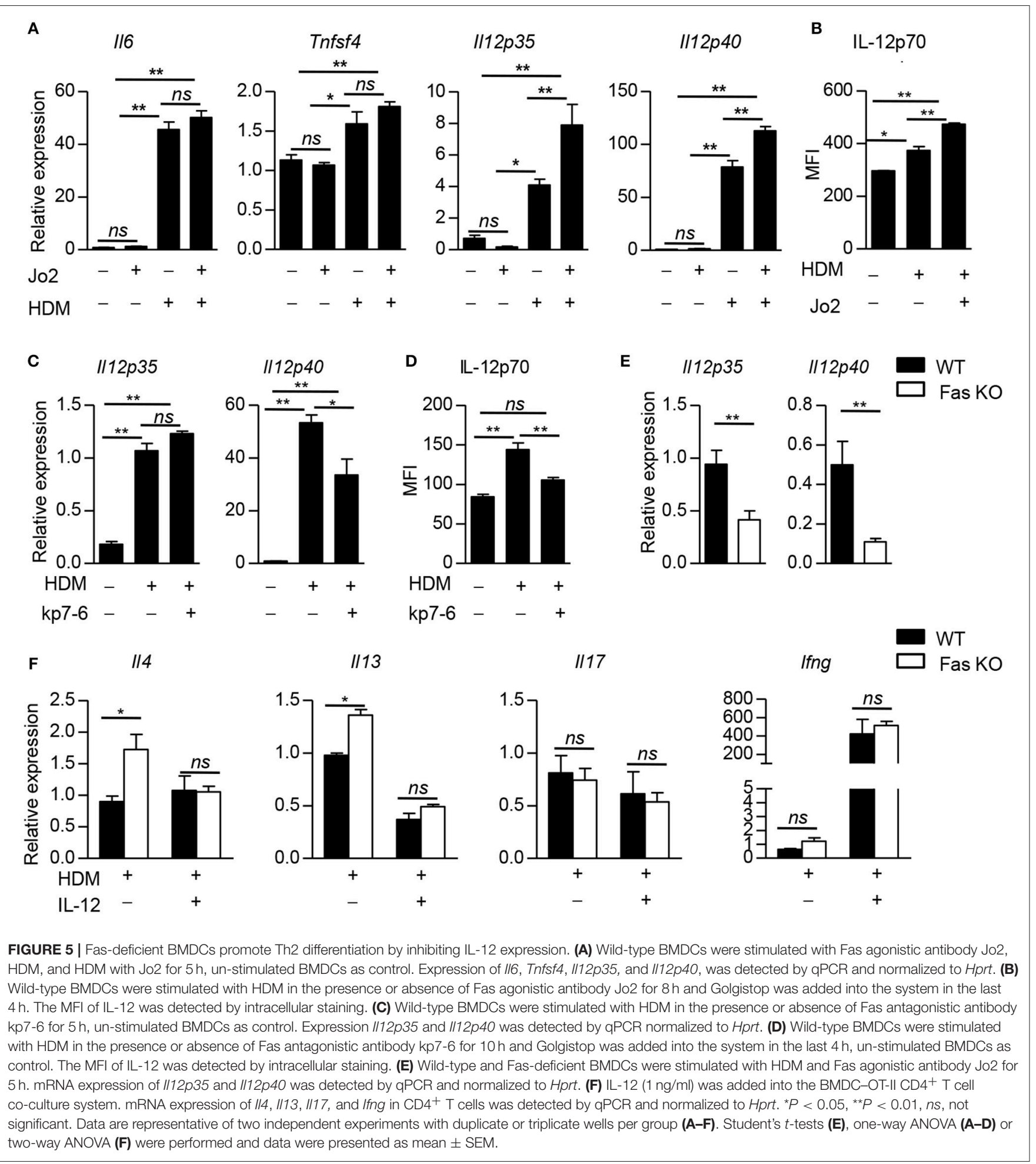

ERK and subsequent IL-1 $\beta$ secretion (34). In the current study, we found that Jo2 stimulation could dramatically decrease the activity of ERK in the presence of HDM. This different role might be caused by the type of stimuli and the status of the BMDCs, and further study need to be explored the potential mechanism for this different regulation by ERK. ERK signaling could profoundly influence the immune response of T cells. ERK activity in $\mathrm{CD}^{+} \mathrm{T}$ cells has a key role in Th2 cell polarization (41). Blocking MEK-ERK signaling effectively suppresses IL12 p40 production from Neospora caninum infected peritoneal 
A

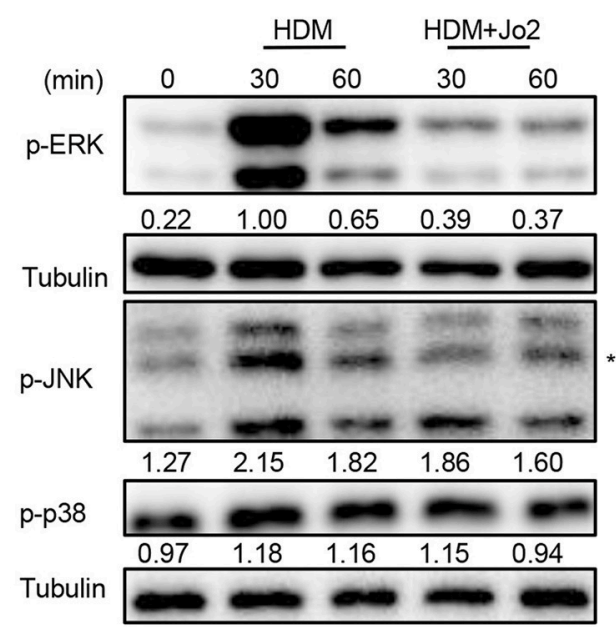

B

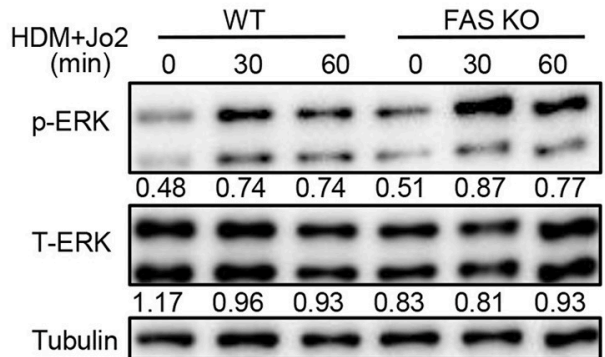

C

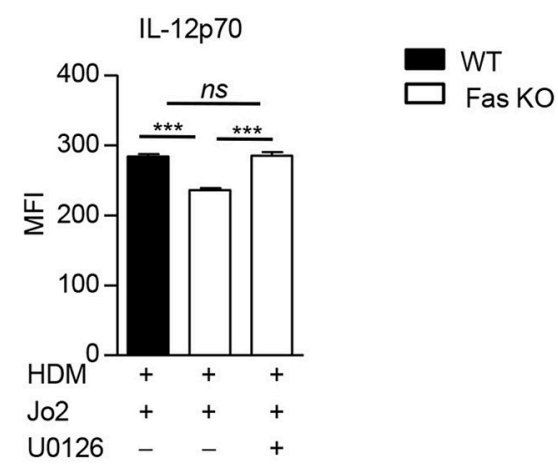

D

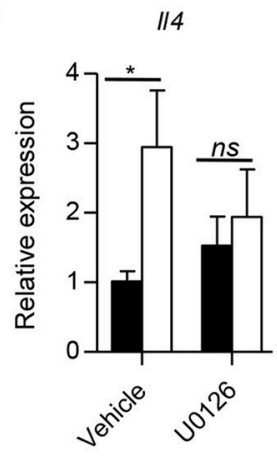

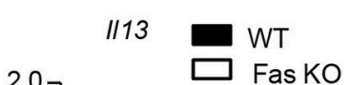

Fas KO

FIGURE 6 | Fas signaling regulates IL-12 expression by modulation of ERK activation in BMDCs. (A) Activity of ERK, JNK, and p38 in wild-type BMDCs stimulated with HDM in the presence or absence of Fas agonistic antibody Jo2 was detected by western blot. Asterisk indicates non-specific bands. Numbers under the lanes indicate band intensity related to that of alpha tubulin. (B) Activation of ERK in wild-type and Fas-deficient BMDCs stimulated with HDM plus Fas agonistic antibody Jo2. Numbers under the lanes indicate band intensity related to that of alpha tubulin. (C) Wild-type and Fas-deficient BMDCs were pre-treated with U0126 for 30 min and then stimulated with HDM and Fas agonistic antibody Jo2 for $8 \mathrm{~h}$. Golgistop was added into the system in the last $4 \mathrm{~h}$. The MFI of IL-12 was detected by intracellular staining. (D) Wild-type and Fas-deficient BMDCs were pre-treated with U0126 for 30 min and then stimulated with HDM for $5 \mathrm{~h}$, washed and co-cultured with OT-II CD4 ${ }^{+}$T cells for $48 \mathrm{~h}$. Expression of $/ / 4$ and $/ / 13$ was detected by qPCR and normalized to Hprt. ${ }^{*} P<0.05$, ${ }^{\star \star \star} P<0.001$, ns, not significant. Data are representative of two independent experiments with duplicate or triplicate wells per group (C) or combination of two experiments with consistent results (D). One-way ANOVA (C) or two-way ANOVA (D) was performed and data were presented as mean $\pm \mathrm{SEM}$.

macrophages (42). In contrast, ERK signaling also has been reported to be an important negative regulator of IL-12 secretion in cigarette smoke extract (CSE) stimulated DCs (43). In this study, we found that a higher ERK activity could suppress IL12 production in HDM-stimulated Fas-deficient BMDCs. Taken together, this study uncovers a specific role of Fas signaling in BMDCs in the regulation of Th2 differentiation and Th2mediated allergic inflammation. Modulation of Fas signaling on DCs may provide a new strategy for treatment of allergic diseases.

\section{AUTHOR CONTRIBUTIONS}

$\mathrm{MH}$ designed and performed the in vivo and cellular experiments and contributed to manuscript writing. $\mathrm{RH}$ performed the in vitro and cellular experiments. JM contributed mouse models. CC contributed to animal colony management. BZ contributed to western blot analysis. HL and JY provided reagents. GH designed experiments, analyzed the data, wrote the manuscript, and provided overall directions.

\section{FUNDING}

This work was supported by the National Natural Science Foundation of China $(81471528,91642104,31670897$ to $\mathrm{GH}$, and 81725004 to HL, 81600788 to MH), the Ministry of Science and Technology of China (973 Basic Science Project 2014CB541803 to $\mathrm{GH}$ ), the Program for Professor of Special Appointment (Eastern Scholar) at Shanghai Institutions of Higher Learning (to $\mathrm{GH})$.

\section{ACKNOWLEDGMENTS}

We thank Dr. Qibin Leng (Institut Pasteur of Shanghai, China) for kindly providing the J5 cell line.

\section{SUPPLEMENTARY MATERIAL}

The Supplementary Material for this article can be found online at: https://www.frontiersin.org/articles/10.3389/fimmu. 2018.03045/full\#supplementary-material 


\section{REFERENCES}

1. Lambrecht BN, Hammad H. The immunology of asthma. Nat Immunol. (2015) 16:45-56. doi: 10.1038/ni.3049

2. van Helden MJ, Lambrecht BN. Dendritic cells in asthma. Curr Opin Immunol. (2013) 25:745-54. doi: 10.1016/j.coi.2013.10.002

3. Chuang YH, Suen JL, Chiang BL. Fas-ligand-expressing adenovirustransfected dendritic cells decrease allergen-specific $\mathrm{T}$ cells and airway inflammation in a murine model of asthma. J Mol Med. (2006) 84:595-603. doi: 10.1007/s00109-006-0047-3

4. Gregory LG, Lloyd CM. Orchestrating house dust mite-associated allergy in the lung. Trends Immunol. (2011) 32:402-11. doi: 10.1016/j.it.2011.06.006

5. Tjota MY, Hrusch CL, Blaine KM, Williams JW, Barrett NA, Sperling AI. Signaling through FcR gamma-associated receptors on dendritic cells drives IL-33-dependent T(H)2-type responses. J Allergy Clin Immun. (2014) 134:706-13.e8. doi: 10.1016/j.jaci.2014.06.013

6. Mayer A, Debuisson D, Denanglaire S, Eddahri F, Fievez L, Hercor M, et al. Antigen presenting cell-derived IL-6 restricts Th2-cell differentiation. Eur J Immunol. (2014) 44:3252-62. doi: 10.1002/eji.201444646

7. Krishnamoorthy N, Oriss TB, Paglia M, Fei M, Yarlagadda M, Vanhaesebroeck $\mathrm{B}$, et al. Activation of $\mathrm{c}$-Kit in dendritic cells regulates $\mathrm{T}$ helper cell differentiation and allergic asthma. Nat Med. (2008) 14:565-73. doi: $10.1038 / \mathrm{nm} 1766$

8. Ito T, Wang YH, Duramad O, Hori T, Delespesse GJ, Watanabe N, et al. TSLP-activated dendritic cells induce an inflammatory $\mathrm{T}$ helper type 2 cell response through OX40 ligand. J Exp Med. (2005) 202:1213-23. doi: $10.1084 / \mathrm{Jem} .20051135$

9. Lambrecht BN, Hammad H. Biology of lung dendritic cells at the origin of asthma. Immunity (2009) 31:412-24. doi: 10.1016/j.immuni.2009.08.008

10. Shibaki A, Katz SI. Activation through CD40 ligation induces functional Fas ligand expression by Langerhans cells. Eur J Immunol. (2001) 31:3006-15. doi: $10.1002 / 1521-4141(2001010) 31: 10 \& \# 60 ; 3006:: A I D-I M M U 3006 \& \# 62 ; 3$. $0 . \mathrm{CO} ; 2-\mathrm{L}$

11. Duez C, Tomkinson A, Shultz LD, Bratton DL, Gelfand EW. Fas deficiency delays the resolution of airway hyperresponsiveness after allergen sensitization and challenge. J Allergy Clin Immunol. (2001) 108:547-56. doi: $10.1067 /$ mai.2001.118288

12. Tong J, Bandulwala HS, Clay BS, Anders RA, Shilling RA, Balachandran DD, et al. Fas-positive $\mathrm{T}$ cells regulate the resolution of airway inflammation in a murine model of asthma. J Exp Med. (2006) 203:1173-84. doi: $10.1084 /$ jem. 20051680

13. Rescigno M, Piguet V, Valzasina B, Lens S, Zubler R, French L, et al. Fas engagement induces the maturation of dendritic cells (DCs), the release of interleukin (IL)-1beta, and the production of interferon gamma in the absence of IL-12 during DC-T cell cognate interaction: a new role for Fas ligand in inflammatory responses. J Exp Med. (2000) 192:1661-8. doi: $10.1084 /$ jem.192.11.1661

14. Meyer Zu Horste G, Przybylski D, Schramm MA, Wang C, Schnell A, Lee Y, et al. Fas promotes T Helper 17 cell differentiation and inhibits T Helper 1 cell development by binding and Sequestering Transcription Factor STAT1. Immunity (2018) 48:556-69 e7. doi: 10.1016/j.immuni.2018.03.008

15. Bien K, Zmigrodzka M, Orlowski P, Fruba A, Szymanski L, Stankiewicz $\mathrm{W}$, et al. Involvement of Fas/FasL pathway in the murine model of atopic dermatitis. Inflamm Res. (2017) 66:679-90. doi: 10.1007/s00011-017-1049-Z

16. Stewart SA, Dykxhoorn DM, Palliser D, Mizuno H, Yu EY, An DS, et al. Lentivirus-delivered stable gene silencing by RNAi in primary cells. RNA (2003) 9:493-501. doi: 10.1261/rna.2192803

17. Harris NL, Watt V, Ronchese F, Le Gros G. Differential T cell function and fate in lymph node and nonlymphoid tissues. J Exp Med. (2002) 195:317-26. doi: 10.1084/jem.20011558

18. Ji WJ, Ma YQ, Zhou X, Zhang YD, Lu RY, Sun HY, et al. Temporal and spatial characterization of mononuclear phagocytes in circulating, lung alveolar and interstitial compartments in a mouse model of bleomycin-induced pulmonary injury. J Immunol Methods (2014) 403:7-16. doi: 10.1016/j.jim.2013.11.012

19. Fulkerson PC, Fischetti CA, Hassman LM, Nikolaidis NM, Rothenberg ME. Persistent effects induced by IL-13 in the lung. Am J Respir Cell Mol Biol. (2006) 35:337-46. doi: 10.1165/rcmb.2005-0474OC
20. Kang Z, Swaidani S, Yin W, Wang C, Barlow JL, Gulen MF, et al. Epithelial cell-specific Actl adaptor mediates interleukin-25-dependent helminth expulsion through expansion of $\operatorname{Lin}(-) \mathrm{c}-\mathrm{Kit}(+)$ innate cell population. Immunity (2012) 36:821-33. doi: 10.1016/j.immuni.2012.03.021

21. Zheng W, Flavell RA. The transcription factor GATA-3 is necessary and sufficient for Th2 cytokine gene expression in CD4 T cells. Cell (1997) 89:58796.

22. Angkasekwinai P, Chang SH, Thapa M, Watarai H, Dong C. Regulation of IL-9 expression by IL-25 signaling. Nat Immunol. (2010) 11:250-6. doi: 10.1038/ni.1846

23. Slawek A, Maj T, Chelmonska-Soyta A. CD40, CD80, and CD86 costimulatory molecules are differentially expressed on murine splenic antigen-presenting cells during the pre-implantation period of pregnancy, and they modulate regulatory $\mathrm{T}$-cell abundance, peripheral cytokine response, and pregnancy outcome. Am J Reprod Immunol. (2013) 70:116-26. doi: 10.1111/aji.12108

24. Bell BD, Kitajima M, Larson RP, Stoklasek TA, Dang K, Sakamoto K, et al. The transcription factor STAT5 is critical in dendritic cells for the development of TH2 but not TH1 responses. Nat Immunol. (2013) 14:364-71. doi: 10.1038/ni.2541

25. Huang G, Wang Y, Vogel P, Kanneganti TD, Otsu K, Chi H. Signaling via the kinase p38alpha programs dendritic cells to drive TH17 differentiation and autoimmune inflammation. Nat Immunol. (2012) 13:152-61. doi: $10.1038 /$ ni.2207

26. Mesnil C, Raulier S, Paulissen G, Xiao X, Birrell MA, Pirottin D, et al. Lungresident eosinophils represent a distinct regulatory eosinophil subset. J Clin Invest. (2016) 126:3279-95. doi: 10.1172/JCI85664

27. Holgate ST. Innate and adaptive immune responses in asthma. Nat Med. (2012) 18:673-83. doi: 10.1038/nm.2731

28. Lamhamedi-Cherradi SE, Martin RE, Ito T, Kheradmand F, Corry DB, Liu YJ, et al. Fungal proteases induce Th2 polarization through limited dendritic cell maturation and reduced production of IL-12. J Immunol. (2008) 180:6000-9. doi: 10.4049/jimmunol.180.9.6000

29. Lambrecht BN, De Veerman M, Coyle AJ, Gutierrez-Ramos JC, Thielemans K, Pauwels RA. Myeloid dendritic cells induce Th2 responses to inhaled antigen, leading to eosinophilic airway inflammation. J Clin Invest. (2000) 106:551-9. doi: 10.1172/JCI8107

30. Shao Z, Bharadwaj AS, McGee HS, Makinde TO, Agrawal DK. Fms-like tyrosine kinase 3 ligand increases a lung DC subset with regulatory properties in allergic airway inflammation. J Allergy Clin Immunol. (2009) 123:917-24.e2. doi: 10.1016/j.jaci.2009.01.052

31. Takagi T, Taguchi O, Toda M, Ruiz DB, Bernabe PG, D’Alessandro-Gabazza $\mathrm{CN}$, et al. Inhibition of allergic bronchial asthma by Thrombomodulin is mediated by dendritic cells. Am J Respir Critic Med. (2011) 183:31-42. doi: 10.1164/rccm.201001-0107OC

32. Na H, Cho M, Chung Y. Regulation of Th2 cell immunity by dendritic cells. Immune Network (2016) 16:1-12. doi: 10.4110/in.2016.16.1.1

33. Guo Z, Zhang M, Tang H, Cao X. Fas signal links innate and adaptive immunity by promoting dendritic-cell secretion of CC and CXC chemokines. Blood (2005) 106:2033-41. doi: 10.1182/blood-2004-12-4831

34. Guo Z, Zhang M, An H, Chen W, Liu S, Guo J, et al. Fas ligation induces IL1beta-dependent maturation and IL-1beta-independent survival of dendritic cells: different roles of ERK and NF-kappaB signaling pathways. Blood (2003) 102:4441-7. doi: 10.1182/blood-2002-11-3420

35. Kataoka T, Budd RC, Holler N, Thome M, Martinon F, Irmler M, et al The caspase- 8 inhibitor FLIP promotes activation of NF-kappaB and Erk signaling pathways. Curr Biol. (2000) 10:640-8. doi: 10.1016/S0960-9822(00) 00512-1

36. van Rijt LS, Vos N, Willart M, Kleinjan A, Coyle AJ, Hoogsteden HC, et al. Essential role of dendritic cell CD80/CD86 costimulation in the induction, but not reactivation, of $\mathrm{TH} 2$ effector responses in a mouse model of asthma. $J$ Allergy Clin Immunol. (2004) 114:166-73. doi: 10.1016/j.jaci.2004.03.044

37. Wu Q, Tang Y, Hu X, Wang Q, Lei W, Zhou L, et al. Regulation of Th1/Th2 balance through OX40/OX40L signalling by glycyrrhizic acid in a murine model of asthma. Respirology (2016) 21:102-11. doi: 10.1111/resp.12655

38. Lin YL, Chen SH, Wang JY. Critical role of IL-6 in dendritic cellinduced allergic inflammation of asthma. J Mol Med. (2015) 94:51-9. doi: $10.1007 /$ s00109-015-1325-8 
39. Laouini D, Alenius H, Bryce P, Oettgen H, Tsitsikov E, Geha RS. IL-10 is critical for Th2 responses in a murine model of allergic dermatitis. J Clin Invest. (2003) 112:1058-66. doi: 10.1172/Jci2003 18246

40. Zhang YL, Zhou XP, Zhou BH. DC-derived TSLP promotes Th2 polarization in LPS-primed allergic airway inflammation. Eur J Immunol. (2012) 42:1735-43. doi: 10.1002/eji.2011 42123

41. Tripathi P, Sahoo N, Ullah U, Kallionpaa H, Suneja A, Lahesmaa R, et al. A novel mechanism for ERK-dependent regulation of IL4 transcription during human Th2-cell differentiation. Immunol Cell Biol. (2012) 90:676-87. doi: $10.1038 /$ icb.2011.87

42. Jin XX, Gong PT, Zhang XC, Li GJ, Zhu T, Zhang MG, et al. Activation of ERK signaling via TLR11 induces IL-12p40 production in peritoneal macrophages challenged by neospora caninum. Front Microbiol. (2017) 8:1393. doi: $10.3389 /$ fmicb. 2017.01393
43. Kroening PR, Barnes TW, Pease L, Limper A, Kita H, Vassall R. Cigarette smoke-induced oxidative stress suppresses generation of dendritic cell IL-12 and IL-23 through ERK-dependent pathways. J Immunol. (2008) 181:1536-47. doi: 10.4049/jimmunol.181.2.1536

Conflict of Interest Statement: The authors declare that the research was conducted in the absence of any commercial or financial relationships that could be construed as a potential conflict of interest.

Copyright (c) $2018 \mathrm{Han}, \mathrm{Hu}, \mathrm{Ma}$, Zhang, Chen, Li, Yang and Huang. This is an open-access article distributed under the terms of the Creative Commons Attribution License (CC BY). The use, distribution or reproduction in other forums is permitted, provided the original author(s) and the copyright owner(s) are credited and that the original publication in this journal is cited, in accordance with accepted academic practice. No use, distribution or reproduction is permitted which does not comply with these terms. 